\title{
Reconsidering the logic of World Federation of Neurosurgical Societies grading in patients with severe subarachnoid hemorrhage
}

\author{
Christian Fung, MD, ${ }^{1}$ Fabienne Inglin, MD, ${ }^{1}$ Michael Murek, MD, ${ }^{1}$ Mathias Balmer, MD, ${ }^{1}$ \\ Janine Abu-Isa, MD, ${ }^{1}$ Werner J. Z'Graggen, MD,, Christoph Ozdoba, MD, ${ }^{2}$ Jan Gralla, MD, MSc, ${ }^{2}$ \\ Stephan M. Jakob, MD, PhD, ${ }^{3}$ Jukka Takala, MD, PhD, ${ }^{3}$ Jürgen Beck, MD, ${ }^{1}$ and Andreas Raabe, MD ${ }^{1}$ \\ ${ }^{1}$ Department of Neurosurgery, ${ }^{2}$ Institute for Diagnostic and Interventional Neuroradiology, and ${ }^{3}$ Department of Intensive Care \\ Medicine, Bern University Hospital (Inselspital) and University of Bern, Switzerland
}

\begin{abstract}
OBJECTIVE Current data show a favorable outcome in up to $50 \%$ of patients with World Federation of Neurosurgical Societies (WFNS) Grade V subarachnoid hemorrhage (SAH) and a rather poor prediction of worst cases. Thus, the usefulness of the current WFNS grading system for identifying the worst scenarios for clinical studies and for making treatment decisions is limited. One reason for this lack of differentiation is the use of "negative" or "silent" diagnostic signs as part of the WFNS Grade V definition. The authors therefore reevaluated the WFNS scale by using "positive" clinical signs and the logic of the Glasgow Coma Scale as a progressive herniation score.

METHODS The authors performed a retrospective analysis of 182 patients with SAH who had poor grades on the WFNS scale. Patients were graded according to the original WFNS scale and additionally according to a modified classification, the WFNS herniation (hWFNS) scale (Grade IV, no clinical signs of herniation; Grade V, clinical signs of herniation). The prediction of poor outcome was compared between these two grading systems.

RESULTS The positive predictive values of Grade V for poor outcome were 74.3\% (OR 3.79, 95\% Cl 1.94-7.54) for WFNS Grade V and $85.7 \%$ (OR 8.27, 95\% Cl 3.78-19.47) for hWFNS Grade V. With respect to mortality, the positive predictive values were $68.3 \%$ (OR 3.9, 95\% Cl 2.01-7.69) for WFNS Grade V and 77.9\% (OR 6.22, 95\% Cl 3.07-13.14) for hWFNS Grade V.

CONCLUSIONS Limiting WFNS Grade V to the positive clinical signs of the Glasgow Coma Scale such as flexion, extension, and pupillary abnormalities instead of including "no motor response" increases the prediction of mortality and poor outcome in patients with severe SAH.

http://thejns.org/doi/abs/10.3171/2015.2.JNS14614
\end{abstract}

KEY WORDS Glasgow Coma Scale; subarachnoid hemorrhage; World Federation of Neurosurgical Societies grading; vascular disorders

$\mathrm{D}$ EATH and severe disability after spontaneous subarachnoid hemorrhage (SAH) are related to the initial severity of the hemorrhage. The scale most often used and recommended is the World Federation of Neurosurgical Societies (WFNS) scale, in which the clinical condition is primarily graded by the Glasgow Coma Scale (GCS). ${ }^{13}$ Despite low GCS scores in poor-grade $\mathrm{SAH}, 35 \%-50 \%$ of aggressively treated patients show a favorable outcome. ${ }^{1,4,12,13,15,18,22}$ Until now there has been no validated triage scheme to identify which patients with SAH will most likely not benefit from aggressive treatment based on WFNS scores. ${ }^{22}$ Even in the most severe SAH (i.e., WFNS Grade V), a good outcome is observed in $24 \%-50 \%$ of patients treated aggressively. ${ }^{1,12,15,32}$

In comatose patients the motor score of the GCS (mGCS) is the best indicator of clinical outcome. ${ }^{22,30} \mathrm{Ac}-$ cording to the original description and the contemporary use of the GCS, patients with an mGCS of 3 or 2 exhibit

ABBREVIATIONS GCS, mGCS = Glasgow Coma Scale, motor score of the GCS; $m$ RS = modified Rankin Scale; PPV = positive predictive value; SAH = subarachnoid hemorrhage; WFNS, hWFNS = World Federation of Neurosurgical Societies, WFNS herniation scale.

ACCOMPANYING EDITORIAL See pp 296-298. DOI: 10.3171/2015.3.JNS15336.

SUBMITTED July 7, 2014. ACCEPTED February 11, 2015.

INCLUDE WHEN CITING Published online September 18, 2015; DOI: 10.3171/2015.2.JNS14614. 
abnormal flexion (decorticate posture) or extension (decerebrate posture), respectively; i.e., they show clinical signs of brainstem dysfunction or herniation..$^{29}$ Although not explicitly mentioned in the usual description of the scale, the GCS was originally created for grading patients with head injury, and the motor score essentially describes a progressive downward brain herniation with clinical signs of decorticate posturing (flexion, GCS score of 5, or mGCS score of 3) followed by decerebrate posturing (extension, GCS score of 4, or mGCS score of 2). Because the motor equivalent for the next stage of progressive herniation is flaccidity, a GCS score of 3 means that the patient shows no motor response. This, however, is a well-known cause of misclassification. ${ }^{27,31}$ Logically, as herniation proceeds, decerebrate posturing (which indicates midbrain or pontine compression) is followed not only by flaccidity but also by signs of medullary compression such as third cranial nerve dysfunction and progressive loss of brainstem reflexes.

The usefulness of including brainstem reflexes and third nerve dysfunction to identify different stages of herniation in comatose patients has been previously shown. ${ }^{31}$ In-hospital mortality was highly associated with signs of herniation. Because the GCS is the basis for WFNS grading, information about progressive herniation beyond the motor response and posturing-e.g., third nerve dysfunction or loss of brainstem reflexes-is unfortunately not incorporated in routine SAH grading. As a known consequence, patients who are sedated and treated with ventilation who do not exhibit a motor response but have symmetrically reactive pupils and intact brainstem reflexes may be wrongly classified as having a GCS score of 3 and thus WFNS Grade V, which leads to inaccuracies in the prognostic power of the WFNS in poor-grade patients.

In this study we applied the concept by following the logic of the original GCS design and the pathophysiological concept behind the scale. During the progression of brain herniation, abnormal flexion (decorticate rigidity, late diencephalic syndrome) is followed by abnormal extensor posturing (decerebrate rigidity, mesencephalic syndrome). Both are "positive" signs; i.e., signs that can be observed by the examiner. If herniation progresses further, damage occurs to the medulla, and the typical signs are flaccidity, no response to pain, mydriasis, and no reaction to light. Using the latter positive signs would help to verify a patient with a true GCS score of 3 .

Our purpose was to reevaluate the prognostic power of the WFNS Grade V classification, particularly with regard to poor-grade patients. We compared the existing WFNS grading system with a modified WFNS herniation grading model (hWFNS) with respect to predicting poor outcome and death.

\section{Methods}

We performed a single-center retrospective analysis that included all patients with spontaneous SAH who were admitted to the Neurosurgical Unit of the Bern University Hospital between January 2005 and December 2010. The local ethics committee approved the study. By thorough chart review we identified GCS scores recorded at first contact with medical personnel, at admission to the neurosurgical unit, and after diversion of CSF. The GCS score after CSF diversion was used for grading the severity of SAH according to the WFNS scale. ${ }^{8}$ If continuous sedation was required for patient care, the last GCS score before intubation was used for WFNS grading. Demographic and clinical data were recorded. Signs of herniation (anisocoric pupils, bilaterally dilated pupils, no corneal reflexes, posturing) were recorded up to the time of aneurysm treatment. Admission imaging was used to assess Fisher grade, ventricular dilation, and intracerebral hemorrhage. ${ }^{7,9} \mathrm{~A}$ CSF shunt was placed if a patient showed clinical and radiological signs of hydrocephalus, defined as decreased level of consciousness and ventricular dilation. In comatose patients an external ventricular drain was placed, which was also used for monitoring intracranial pressure. Patients were treated according to standard SAH guidelines, which included early angiography and transfer to an intensive care unit. ${ }^{6}$ Aggressive treatment of poor-grade patients was at the discretion of the treating surgeon and was based on parameters like clinical presentation, age, pretreatment morbidities, and the patient's expressed wishes. Outcome was assessed according to the modified Rankin Scale (mRS) at 6 months post-SAH, when patients were hospitalized for a control angiography, or during visits to the outpatient clinic. Outcome was dichotomized into good (mRS Score 0-3) and poor (mRS Score 4-6).

\section{The WFNS and hWFNS Grading Models}

Patients were graded according to the original WFNS scale, in which GCS Score 3-6 corresponds to WFNS Grade V and GCS Score 7-12 corresponds to WFNS Grade IV.

To test our hypothesis, these patients were also graded according to our modified WFNS classification, the hWFNS. In this modification of Grade V, we only included patients with signs of flexion or extension or positive clinical signs of brainstem dysfunction (anisocoric pupils, bilaterally dilated pupils, no corneal reflexes). Thus, patients with a GCS score of 6-12 or those with no motor response but otherwise intact brainstem function were graded hWFNS Grade IV. Thus, a patient graded as GCS 3 (no motor response) but with 3-mm symmetrical pupils with prompt reaction to light and intact corneal reflex was assigned hWFNS Grade IV instead of WFNS Grade V. In summary, contrary to patients with hWFNS IV, those with hWFNS V showed at least one of the following signs of brainstem dysfunction or herniation: anisocoric pupils, bilaterally dilated pupils, no corneal reflexes, or posturing (flexion, extension). The two classification methods were compared with respect to predicting poor outcome and death.

\section{Statistical Analysis}

Comparison between the WFNS and hWFNS grading systems was done using OR, positive predictive value (PPV), sensitivity, and specificity. The OR and PPV for poor outcomes were calculated using contingency tables. The 95\% CIs for ORs were obtained according to the efficient-score method. ${ }^{20}$ The Fisher exact test was used to calculate 2 -sided $\mathrm{p}$ values. We also created likelihood ra- 
tio graphs, with which the difference in diagnostic quality of different scales can be read by the relative position of their likelihood ratio graphs. ${ }^{5,7}$

\section{Results}

This retrospective analysis included 428 patients with spontaneous SAH who were admitted to the Neurosurgical Department of the Bern University Hospital between January 2005 and December 2010. We identified 182 patients with poor-grade $\mathrm{SAH}$, of whom 80 were assigned WFNS Grade IV and 102 were WFNS Grade V. The study included 56 men and 126 women (ratio 1:2.3), with a median age of 60 years (interquartile range 53-69 years). According to initial imaging, 6 patients $(3.3 \%)$ were Fisher Grade 2, 137 (75.3\%) were Fisher Grade 3, and 39 (21.4\%) were Fisher Grade 4. One hundred sixty-four patients (90.1\%) had intraventricular and/or intraparenchymatous hemorrhage. Thirty-eight patients (20.9\%) received aneurysm clipping and $67(36.8 \%)$ received coil intervention. Two patients were lost to follow-up. The distribution of patients according to the two different WFNS classifications and outcome parameters is displayed in Table 1. Both WFNS scores showed a highly significant association with respect to mortality and outcome at 6 months. According to the two classification methods, 101 patients (WFNS) and 77 patients (hWFNS) were assigned SAH Grade V. According to the WFNS classification, 75 of 101 patients with Grade V had a poor outcome after 6 months, which resulted in a PPV for poor outcome of $74.3 \%$ (OR $3.79,95 \%$ CI 1.94-7.54) (Table 1, Fig. 1). According to the hWFNS classification, 66 of 77 patients with Grade V had a poor outcome: PPV $85.7 \%$ (OR 8.27, 95\% CI 3.78-19.47).

Sixty-nine of 101 patients with WFNS Grade V died: PPV 68.3\% (OR 3.9, 95\% CI 2.01-7.69), compared with 60 of 77 patients with hWFNS Grade V: PPV 77.9\% (OR $6.22,95 \%$ CI 3.07-13.14). Figure 1 shows that the hWFNS scale has an overall better diagnostic quality with respect to poor outcome. With respect to mortality after 6 months, the two scales (WFNS and hWFNS) are equal (Fig. 2).

\section{Discussion}

\section{The Pathophysiological Concept and Logic Behind the Lower GCS Scores}

The GCS was originally designed to classify different states of impaired consciousness. ${ }^{29}$ Impaired conscious- ness as an expression of brain dysfunction can be caused by focal or diffusely acting agents. Supratentorial lesions can cause impaired consciousness by two major clinical herniation syndromes: the uncal herniation syndrome causes an early third nerve compression and a lateral midbrain compression, and the central herniation syndrome begins with bilateral diencephalic impairment. Although they have different starting points, if herniation progresses both syndromes proceed to a final stage of mesencephalic and upper medullary involvement. During central herniation in its late diencephalic stage, stretching and compression of feeding vessels and impairment of the ascending arousal system causes coma, flexor posturing (decorticate posturing), and yet small reactive pupils. ${ }^{21}$ The diencephalic stage is followed by the midbrain stage, where pupils become fixed at midposition. Motor responses show extensor posturing (decerebrate posturing), either spontaneously or in response to stimuli. Extensor posturing is believed to be due to a release of vestibulospinal postural reflexes from forebrain control, ${ }^{21}$ and the fixed position of the pupils is presumably caused by central impairment of both the sympathetic and parasympathetic central pathways. Next the patient enters the pontine stage, where motor tone becomes flaccid, pupils dilated and irregular, and breathing irregular and shallow due to destruction of upper pontine structures. Finally, in the medullary stage, the pupils are maximally dilated and breathing fails, with no chance of useful recovery. ${ }^{21}$

In early uncal herniation the pupil is dilated but still reacts to light, which is abolished in the late uncal herniation syndrome..$^{21,25}$ Pupillary dilation in uncal herniation syndrome is caused, in contrast to the central herniation syndrome, by direct compression of the oculomotor nerve by herniated tissue. ${ }^{23}$ As uncal herniation proceeds, the next stage is the midbrain stage (see above), skipping the diencephalic stage. With progressive herniation, the subsequent stages are equivalent to the central herniation syndrome. Including this pathophysiology of progressive herniation in the current WFNS grading system, our data show that discrimination and outcome predictions improve when clinical signs of brainstem dysfunction are used as additional criteria for assigning a GCS score of 3 .

\section{Weakness of the Current WFNS Grade V Definition}

The so-called brainstem sign modification avoids labeling patients who might actually have a better prognosis

TABLE 1. Outcome at 6 months according to the mRS: comparison of WFNS and hWFNS scales

\begin{tabular}{|c|c|c|c|c|c|c|c|c|c|c|}
\hline \multirow[b]{2}{*}{ Scale } & \multicolumn{2}{|c|}{ Outcome* } & \multirow{2}{*}{$\begin{array}{l}\text { PPV } \\
(\%) \dagger\end{array}$} & \multirow[b]{2}{*}{ Sensitivity $(95 \% \mathrm{CI})$} & \multirow[b]{2}{*}{ Specificity $(95 \%$ Cl) } & \multirow[b]{2}{*}{ Alive } & \multirow[b]{2}{*}{ Dead } & \multirow{2}{*}{$\begin{array}{l}\text { PPV } \\
(\%) \dagger\end{array}$} & \multirow[b]{2}{*}{ Sensitivity $(95 \% \mathrm{Cl})$} & \multirow[b]{2}{*}{ Specificity $(95 \% \mathrm{Cl})$} \\
\hline & Good & Poor & & & & & & & & \\
\hline \multicolumn{11}{|l|}{ WFNS } \\
\hline Grade IV & 45 & 34 & & & & 51 & 28 & & & \\
\hline Grade V & 26 & 75 & 74.3 & $0.69(0.59-0.77)$ & $0.63(0.51-0.75)$ & 32 & 69 & 68.3 & $0.71(0.61-0.8)$ & $0.61(0.5-0.72)$ \\
\hline \multicolumn{11}{|l|}{ hWFNS } \\
\hline Grade IV & 60 & 43 & & & & 66 & 37 & & & \\
\hline Grade V & 11 & 66 & 85.7 & $0.61(0.51-0.7)$ & $0.85(0.74-0.92)$ & 17 & 60 & 77.9 & $0.62(0.51-0.72)$ & $0.8(0.7-0.9)$ \\
\hline
\end{tabular}



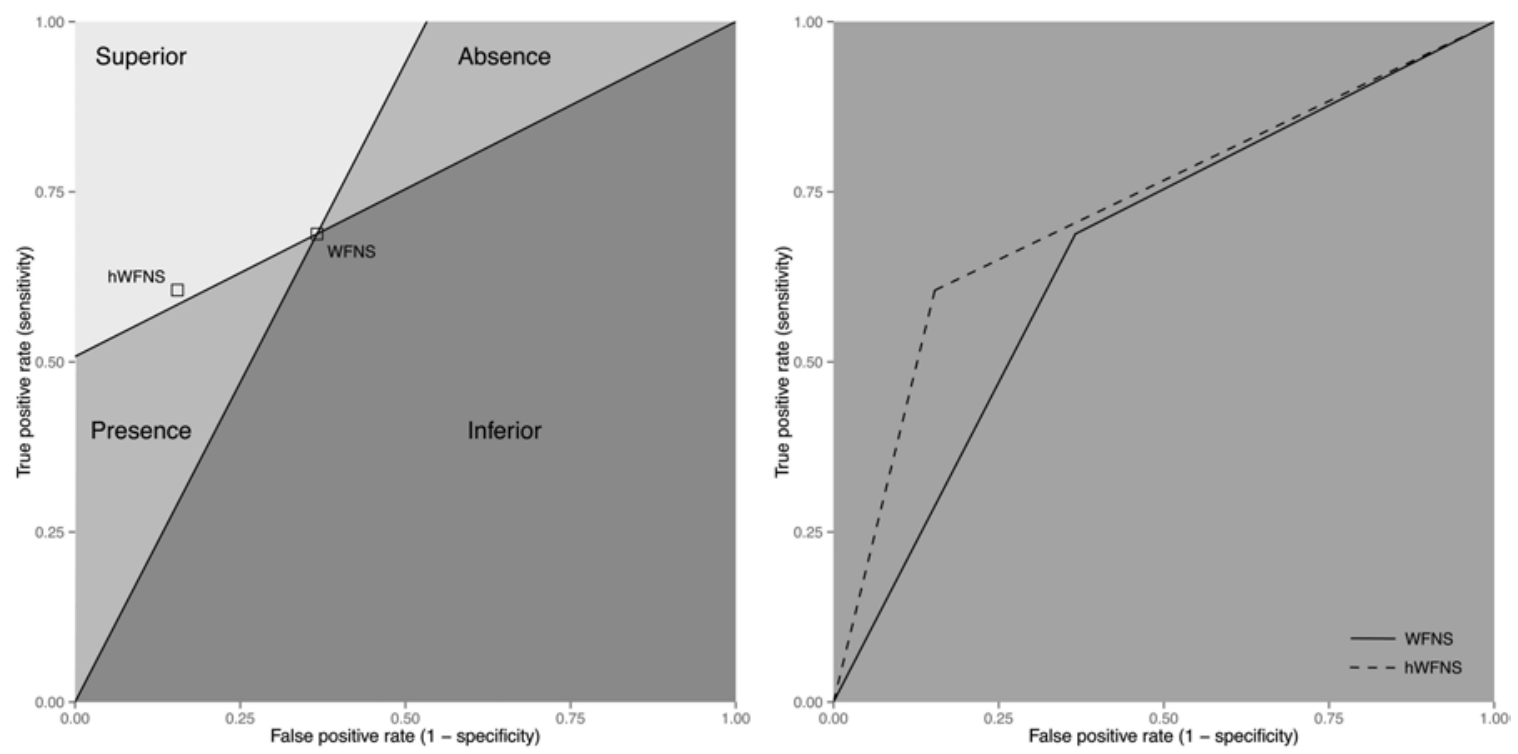

FIG. 1. Likelihood ratio graphs comparing the diagnostic value of the WFNS and hWFNS grades with respect to poor outcome. The graphs are created by plotting " 1 - specificities" (false-positive rate) against "sensitivities" (true-positive rate) and drawing lines through these points and the coordinates $(0,0)$ and $(1,1)$. The slope of the line through $(0,0)$ is equal to the likelihood ratio of a positive test, where steeper slopes indicate greater diagnostic ability. The slope of the line through $(1,1)$ equals the likelihood ratio of a negative test. Less steep slopes indicate greater diagnostic ability. Left: Graph showing regions of comparison: compared with WFNS, a diagnostic test in the "Superior" region (hWFNS) is superior overall; the "Absence" region is superior for confirming absence of poor outcome; the "Presence" region is superior for confirming presence of poor outcome; and the "Inferior" region is inferior overall. Right: Likelihood ratio graph of WFNS and hWFNS for poor outcome.

but are included in the original WFNS Grade V with a GCS score of 6 or a misclassified GCS score of 3. Comparable results have been published for mGCS in patients with poor-grade SAH who do not improve above an mGCS score of $3,{ }^{30}$ and for prediction of morbidity and mortality in childhood drowning or near-drowning. ${ }^{10,14} \mathrm{In}$ these cases, unreactive pupils, decorticate, decerebrate, or flaccid posture (GCS score $\leq 5$ ) were the best independent predictors of poor neurological outcome and death. ${ }^{10,14}$

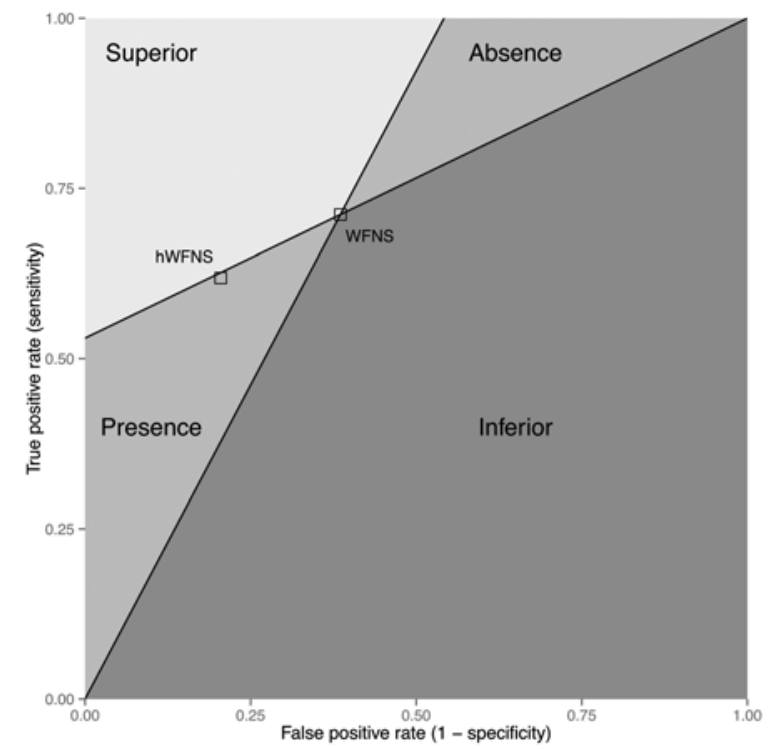

Although diffuse ischemic or hypoxic brain damage after SAH is less reliably graded with the GCS, this flaw applies more to the original WFNS scale, because the hWFNS scale incorporates these signs.

Major shortcomings of the GCS score are as follows: 1) the difficulty in assessing verbal response in intubated patients; 2) a "missing sign"-no motor response-to allocate the worst grade, which is of poor specificity and is not a pathological finding per se; and 3) the lack of attention

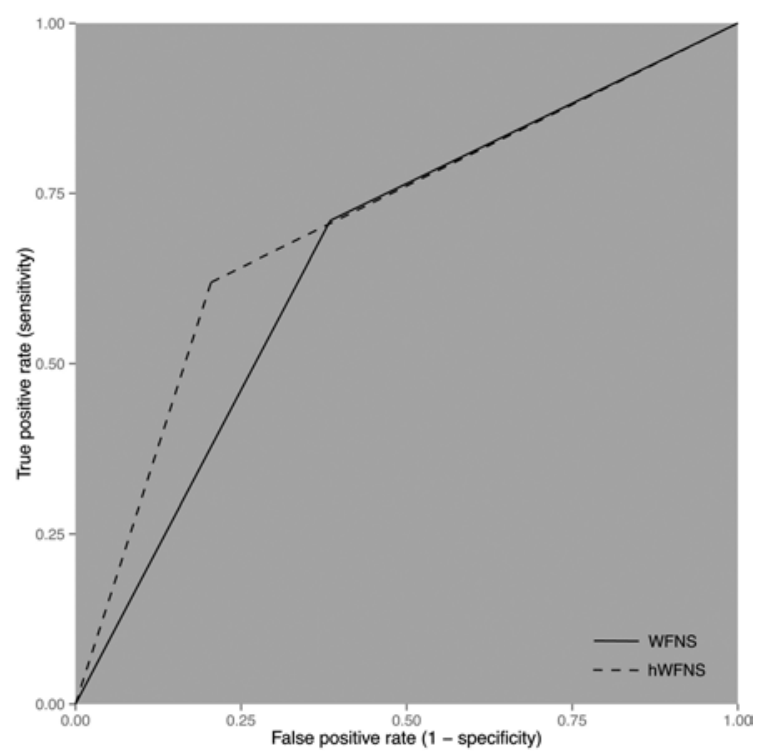

FIG. 2. Left: Likelihood ratio graph comparing the diagnostic value of the WFNS and hWFNS grades with respect to mortality. Right: Likelihood ratio graph of WFNS and hWFNS for mortality. 
to abnormal brainstem reflexes and signs of herniation..$^{27,31}$ The degree of oculomotor dysfunction has even been described as the most valuable indicator of the progress and extent of the herniation. ${ }^{28}$ In addition, recent publications on traumatic brain injury have pointed to the problem of early intubated patients and reliable GCS assessment ${ }^{19}$ as well as the lack of an association between GCS score and outcome. ${ }^{2}$ Besides the shortcomings of the GCS, the clinical status of patients with poor-grade SAH and the quality of assessments can be obscured by intubation, sedation, hydrocephalus, or seizures. ${ }^{3,11,16,17,23}$ In summary, these factors might negatively influence the predictive value of the WFNS grade.

\section{Modification of WFNS Grade V}

Use of the hWFNS scale improved the predictive value of the original WFNS grading. The hWFNS Grade V is based on clearly defined positive pathological findings; i.e., visible signs of herniation or brainstem dysfunction. Using positive signs augments the level of differentiation between WFNS Grades IV and V. Comparable results were presented in earlier studies. . $^{16,26,30}$

Van den Berg et al. showed that neurological improvement $>$ GCS Score 5 (which corresponds to hWFNS Grade IV) is associated with favorable outcome in $39 \%$ of patients, whereas those without improvement (which corresponds to hWFNS Grade V) showed a worse outcome. ${ }^{30}$ Starke et al. showed that each point increase in motor examination score predicted a 1.8-fold increased odds of favorable outcome. ${ }^{26}$ Both methods reflect signs for a beneficial outcome and, according to both methods, a good outcome can only be predicted after clinical improvement (e.g., improvement in mGCS score) either has or has not taken place up to a certain point in time. Mack et al. provided evidence for a clinical sign that predicted negative outcome. ${ }^{16}$ They showed a better mid- and long-term recovery in patients who had intact pupillary reactivity compared with those with uni- or bilateral nonreactive pupils. The hWFNS scale, as in the latter study, predicts poor outcome using only positive clinical signs, which includes posturing (mGCS Scores 2 and 3 ) in its grading scale.

The WFNS scale is widely accepted and our modification would only affect the definition of poor-grade patients (i.e., Grades IV and V). The acceptance of a modification of an existing grading system is not predictable, but mainly depends on the following features: 1) its simplicity; 2) its pathophysiological logic; 3) its accuracy in describing a patient's clinical status; and 4) its relevance for clinical management. The presence of a brainstem sign often has management consequences. Most neurosurgeons already use brainstem signs, when present, in addition to a WFNS Grade V diagnosis when reporting about a patient's neurological status or when deciding about the individual management of a patient.

To validate the findings of this retrospective study, we have initiated a multicenter prospective trial that will start patient recruitment in 2015 (ClinicalTrials.gov, registration no. NCT02304328).

\section{Conclusions}

Demanding positive signs of brainstem herniation or dysfunction before assigning a GCS score of 3 in patients with poor-grade SAH helps to identify patients with "true" WFNS Grade V, and significantly improves prediction of outcome at 6 months. These criteria could easily be implemented into the WFNS grading system.

\section{References}

1. Bailes JE, Spetzler RF, Hadley MN, Baldwin HZ: Management morbidity and mortality of poor-grade aneurysm patients. J Neurosurg 72:559-566, 1990

2. Balestreri M, Czosnyka M, Chatfield DA, Steiner LA, Schmidt EA, Smielewski P, et al: Predictive value of Glasgow Coma Scale after brain trauma: change in trend over the past ten years. J Neurol Neurosurg Psychiatry 75:161-162, 2004

3. Bederson JB, Connolly ES Jr, Batjer HH, Dacey RG, Dion JE, Diringer MN, et al: Guidelines for the management of aneurysmal subarachnoid hemorrhage: a statement for healthcare professionals from a special writing group of the Stroke Council, American Heart Association. Stroke 40:994-1025, 2009

4. Bergui M, Bradac GB: Acute endovascular treatment of ruptured aneurysms in poor-grade patients. Neuroradiology 46:161-164, 2004

5. Biggerstaff BJ: Comparing diagnostic tests: a simple graphic using likelihood ratios. Stat Med 19:649-663, 2000

6. Connolly ES Jr, Rabinstein AA, Carhuapoma JR, Derdeyn CP, Dion J, Higashida RT, et al: Guidelines for the management of aneurysmal subarachnoid hemorrhage: a guideline for healthcare professionals from the American Heart Association/American Stroke Association. Stroke 43:1711-1737, 2012

7. Dankbaar JW, de Rooij NK, Velthuis BK, Frijns CJM, Rinkel GJE, van der Schaaf IC: Diagnosing delayed cerebral ischemia with different CT modalities in patients with subarachnoid hemorrhage with clinical deterioration. Stroke 40:3493-3498, 2009

8. Drake CG (ed): Report of World Federation of Neurological Surgeons committee on a universal subarachnoid hemorrhage grading scale. J Neurosurg 68:985-986, 1988 (Letter)

9. Fisher CM, Kistler JP, Davis JM: Relation of cerebral vasospasm to subarachnoid hemorrhage visualized by computerized tomographic scanning. Neurosurgery 6:1-9, 1980

10. Habib DM, Tecklenburg FW, Webb SA, Anas NG, Perkin RM: Prediction of childhood drowning and near-drowning morbidity and mortality. Pediatr Emerg Care 12:255-258, 1996

11. Hart RG, Byer JA, Slaughter JR, Hewett JE, Easton JD: Occurrence and implications of seizures in subarachnoid hemorrhage due to ruptured intracranial aneurysms. Neurosurgery 8:417-421, 1981

12. Haug T, Sorteberg A, Finset A, Lindegaard KF, Lundar T, Sorteberg W: Cognitive functioning and health-related quality of life 1 year after aneurysmal subarachnoid hemorrhage in preoperative comatose patients (Hunt and Hess Grade V patients). Neurosurgery 66:475-485, 2010

13. Hijdra A, van Gijn J, Nagelkerke NJ, Vermeulen M, van Crevel H: Prediction of delayed cerebral ischemia, rebleeding, and outcome after aneurysmal subarachnoid hemorrhage. Stroke 19:1250-1256, 1988

14. Lavelle JM, Shaw KN: Near drowning: is emergency department cardiopulmonary resuscitation or intensive care unit cerebral resuscitation indicated? Crit Care Med 21:368-373, 1993

15. Le Roux PD, Elliott JP, Newell DW, Grady MS, Winn HR: Predicting outcome in poor-grade patients with subarachnoid hemorrhage: a retrospective review of 159 aggressively managed cases. J Neurosurg 85:39-49, 1996 
16. Mack WJ, Hickman ZL, Ducruet AF, Kalyvas JT, Garrett MC, Starke RM, et al: Pupillary reactivity upon hospital admission predicts long-term outcome in poor grade aneurysmal subarachnoid hemorrhage patients. Neurocrit Care 8:374-379, 2008

17. Mehta V, Holness RO, Connolly K, Walling S, Hall R: Acute hydrocephalus following aneurysmal subarachnoid hemorrhage. Can J Neurol Sci 23:40-45, 1996

18. Mocco J, Ransom ER, Komotar RJ, Schmidt JM, Sciacca $\mathrm{RR}$, Mayer SA, et al: Preoperative prediction of long-term outcome in poor-grade aneurysmal subarachnoid hemorrhage. Neurosurgery 59:529-538, 2006

19. Moskopp D, Stähle C, Wassmann H: Problems of the Glasgow Coma Scale with early intubated patients. Neurosurg Rev 18:253-257, 1995

20. Newcombe RG: Two-sided confidence intervals for the single proportion: comparison of seven methods. Stat Med 17:857872, 1998

21. Plum F, Posner JB: The diagnosis of stupor and coma. Contemp Neurol Ser 10:1-286, 1972

22. Quigley MR, Salary M: Defining survivorship after highgrade aneurysmal subarachnoid hemorrhage. Surg Neurol 69:261-265, 2008

23. Reid WL, Cone WV: The mechanism of fixed dilatation of the pupil-resulting from ipsilateral cerebral compression. JAMA 112:2030-2034, 1939

24. Rhoney DH, Tipps LB, Murry KR, Basham MC, Michael DB, Coplin WM: Anticonvulsant prophylaxis and timing of seizures after aneurysmal subarachnoid hemorrhage. Neurology 55:258-265, 2000

25. Ropper AH: Lateral displacement of the brain and level of consciousness in patients with an acute hemispheral mass. $\mathbf{N}$ Engl J Med 314:953-958, 1986

26. Starke RM, Komotar RJ, Otten ML, Schmidt JM, Fernandez LD, Rincon F, et al: Predicting long-term outcome in poor grade aneurysmal subarachnoid haemorrhage patients utilising the Glasgow Coma Scale. J Clin Neurosci 16:26-31, 2009

27. Sternbach GL: The Glasgow Coma Scale. J Emerg Med 19:67-71, 2000

28. Sunderland S: The tentorial notch and complications produced by herniations of the brain through that aperture. Br J Surg 45:422-438, 1958
29. Teasdale G, Jennett B: Assessment of coma and impaired consciousness. A practical scale. Lancet 2:81-84, 1974

30. van den Berg R, Foumani M, Schröder RD, Peerdeman SM, Horn J, Bipat S, et al: Predictors of outcome in World Federation of Neurologic Surgeons grade V aneurysmal subarachnoid hemorrhage patients. Crit Care Med 39:2722-2727, 2011

31. Wijdicks EF, Bamlet WR, Maramattom BV, Manno EM, McClelland RL: Validation of a new coma scale: The FOUR score. Ann Neurol 58:585-593, 2005

32. Wostrack M, Sandow N, Vajkoczy P, Schatlo B, Bijlenga P, Schaller K, et al: Subarachnoid haemorrhage WFNS grade V: is maximal treatment worthwhile? Acta Neurochir (Wien) 155:579-586, 2013

\section{Disclosure}

The authors report no conflict of interest concerning the materials or methods used in this study or the findings specified in this paper.

\section{Author Contributions}

Conception and design: Raabe, Fung, Beck. Acquisition of data: Fung, Inglin, Balmer, Abu-Isa, Z'Graggen, Ozdoba, Gralla, Jakob, Takala, Beck. Analysis and interpretation of data: Raabe, Fung, Murek, Balmer, Beck. Drafting the article: Raabe, Fung, Beck. Critically revising the article: Raabe, Fung, Murek, Balmer, Abu-Isa, Z'Graggen, Ozdoba, Gralla, Jakob, Takala, Beck. Reviewed submitted version of manuscript: Raabe, Fung, Murek, Balmer, Abu-Isa, Z'Graggen, Ozdoba, Gralla, Jakob, Takala, Beck. Approved the final version of the manuscript on behalf of all authors: Raabe. Statistical analysis: Fung, Murek. Administrative/technical/material support: Inglin. Radiological evaluation: Ozdoba, Gralla.

\section{Correspondence}

Andreas Raabe, Department of Neurosurgery, Bern University Hospital, Freiburgstrasse 10, 3010 Bern, Switzerland. email: andreas.raabe@insel.ch. 